\title{
High strain rate behaviour of fiber reinforced concrete
}

\author{
Miloslav Popovič ${ }^{1, *}$, Jaroslav Buchar ${ }^{1}$, and Martina Drdlová $^{2}$ \\ ${ }^{1}$ SVS FEM s.r.o., Škrochova 42, 615 00, Brno, Czech Republic \\ ${ }^{2}$ Výzkumný ústav stavebních hmot, a. s., Hněvkovského 65, 61700 Brno, Czech Republic
}

\begin{abstract}
The results of dynamic compression and tensile-splitting tests of concrete reinforced by randomly distributed short non - metallic fibres are presented. A Split Hopkinson Pressure Bar combined with a high-speed photographic system, was used to conduct dynamic Brazilian tests. Quasi static test show that the reinforcement of concrete by the non-metallic fibres leads to the improvement of mechanical properties at quasi static loading. This phenomenon was not observed at the high strain rate loading . Some explanation of this result is briefly outlined.
\end{abstract}

\section{Introduction}

The concrete is mainly reinforced by using of steel fibres. It is well known that short discrete steel fibres lead to significant improvement of properties for impact resistance like higher tensile strength, ductility and toughness as compared to plain cementitious matrices [1].

The aim of this paper consists in an experimental study of the influence of non-metallic fibres both on quasi static and dynamic mechanical properties. The concrete specimens with carbon, aramide, glass, polypropylene and wollastonite fibres have been tested.

The response of the given materials to the quasistatic compressive and flexural loading has been studied. The dynamic loading was realized using of the Hopkinson split pressure bar technique (HSBT). Two kinds of dynamic tests were carried out: a classical compressive test and tensile - splitting test (Brazilian test).

Results show that the influence of the fibre reinforcement leads to the improvement in the mechanical properties at the quasi - static loading. The effect of these fibres at the dynamic loading is quite different. Some reasons of this phenomenon are briefly discussed.

\section{Material and Experimental Technique}

The cement used in this work is ordinary Portland cement $52.5 \mathrm{R}$. A superplasticizer is used to adjust the workability of concrete. A micro-sand in the $0-1 \mathrm{~mm}$ range was used. Additionally, three types of fibres are used:

- Aramid fibres with length of $1 \mathrm{~mm}$. Their volume content is $1.5 \%$. Specimens are denoted as SIFAR $1.5 \%$.
- Carbon fibres with length of $3 \mathrm{~mm}$. Their volume content is $1.5 \%$. Specimens are denoted as SIFC $1.5 \%$.

- Recycled glass laminate fibres length $<0.3 \mathrm{~mm}$. Their volume content is $3 \%$. Specimens are denoted as SIFREC3\%.

- Polypropylene fibres, length $3 \mathrm{~mm}$. Their volume content is $1.5 \%$. Specimens denoted as SIFPP $1.5 \%$

- Wollastonite fibres, length $<0.3 \mathrm{~mm}$. Their volume content is $2 \%$. Specimens are denoted as SIFWOL2\%.

The mechanical parameters - compressive and flexural strength were obtained using universal strength testing machine TIRAtest 2710, R58/02. The compressive and flexural load was applied in quasi-static conditions at the speed of $5 \mathrm{~mm} / \mathrm{min}$. The testing works were performed at room temperature of $20^{\circ} \mathrm{C}$ and relative humidity $55 \%$.

The dynamic mechanical properties were obtained using of the Hopkinson Split Pressure Bar technique (HSPBT). This method consists of an incident bar and a transmitter bar, with a short specimen placed between them and a striker bar that produces an impact on the incident bar to generate a longitudinal compressive pulse, $\sigma_{I}(t)$ that propagates toward the specimen. The pulse is partially reflected in the border of the incident bar, stress pulse $\sigma_{\mathrm{R}}(\mathrm{t})$ and partially transmitted through the specimen as the stress pulse $\sigma_{\mathrm{T}}(\mathrm{t})$. The recorded stress pulses enable to evaluate strain, strain rate and stress in the specimen [2]. The maximum of the transmitted stress pulse, $\sigma_{\mathrm{Tm}}$, corresponds to compressive strength of the specimen material. The used specimens had the form of the cylinders, whose diameter and thickness were 15 and $7 \mathrm{~mm}$ respectively.

\footnotetext{
* Corresponding author: mpopovic@svsfem.cz
} 
The same experimental arrangement can be also used for the evaluation of the tensile - splitting strength. The position of the specimen is shown in the Fig.1.

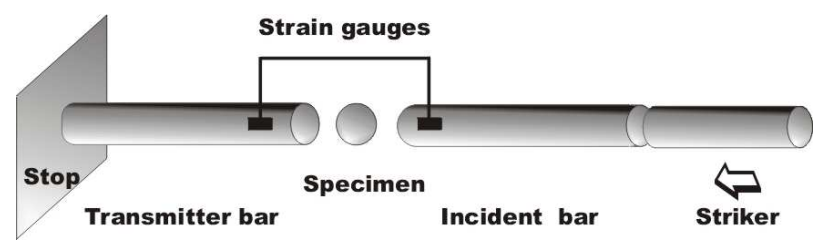

Fig.1. Experimental arrangement of the dynamic tensile - split test (Brazilian test).

This arrangement, which is known as the Brazilian test, or diametric compression test, is a typical indirect tensile test, which was developed to determine the tensile strength of brittle materials such as rock, ceramics and concrete [3-6]. Even this method was developed for the study of the fracture behaviour at quasi-static loading, numerical analysis, Antoun et al. [7] found that the stress distribution in the dynamic Brazilian test was similar to the static test; therefore, they thought that the dynamic Brazilian test was an effective method to measure the tensile properties.

In this case the diametric loading generates tension perpendicular to the load plane, which eventually causes the specimen to split. The strain records of the incident, reflected and transmitted pulses are used to calculate the corresponding stress pulses and the tensile stress in the loading plane, which derives from the following equation

$$
\sigma=\frac{2 P}{\pi W D}
$$

where $\mathrm{P}$ is the load transmitted through the cylinder, $\mathrm{W}$ and $\mathrm{D}$ are respectively the width and diameter of the cylinder. $\mathrm{P}$ is in turn calculated from the transmitted stress. The dynamic tensile strength, $\mathrm{f}_{\mathrm{td}}$, is obtained using Eq. (1) for the maximum transmitted load. The strain rate follows as

$$
\dot{\varepsilon}=\frac{1}{E} \frac{\delta \sigma}{\delta t}
$$

The force $\mathrm{P}$ is obtained using of the recorded stress pulses mentioned above as [2]:

$$
P(t)=\frac{\pi R^{2}}{2}\left[\sigma_{I}(t)+\sigma_{R}(t)+\sigma_{T}\left(t+t_{0}\right)\right]
$$

where $\mathrm{R}$ is the radius of the SHPB and $t_{0}$ is the transition time of the stress wave in the specimen.

Two series of cylindrical specimens have been used. The specimens with $\mathrm{D}=15 \mathrm{~mm}$ and $\mathrm{W}=7 \mathrm{~mm}$ have been used. The second series involved specimens with $\mathrm{D}=30$ $\mathrm{mm}$ and $\mathrm{W}=15 \mathrm{~mm}$. These specimens were used in tests where the course of the Brazilian test was recorded using a high speed camera. For brittle materials, the time from the deformation to the final failure under dynamic loading is very short. To obtain a detailed dynamic deformation and fracture process of the brittle specimens in the dynamic Brazilian test, using a high-speed camera, requires a high frame rate. For a high-speed camera, there is a compromise between the frame rate and image resolution. The fracture of the specimens during the Brazilian test was recorded using the high speed photography (160000 frames per second). Preliminary investigation of the fracture surfaces of broken specimens was also performed.

\section{Results and discussion}

Experimental results on the mechanical properties of the tested materials under quasi - static loading are presented in the Table 1.

Table 1. Quasi - static mechanical properties of the tested materials. $\left(\rho\right.$ is the material density, $\sigma_{\mathrm{c}}$ is the compressive strength, $\sigma_{\mathrm{fc}}$ is the tensile strength).

\begin{tabular}{|c|c|c|c|}
\hline MATERIAL & $\begin{array}{c}\rho \\
\left(\mathrm{kg} . \mathrm{m}^{-3}\right)\end{array}$ & $\begin{array}{c}\sigma_{\mathrm{c}} \\
(\mathrm{MPa})\end{array}$ & $\begin{array}{c}\sigma_{\mathrm{fc}} \\
(\mathrm{MPa})\end{array}$ \\
\hline $\begin{array}{c}\text { SIFCON } \\
\text { (Matrix) }\end{array}$ & 2177 & 73.67 & 9.69 \\
\hline SIF PP 1.5\% & 1911 & 99.51 & 8.74 \\
\hline SIF woll 2\% & 2164 & 104.0 & 12.59 \\
\hline SIF AR 15\% & 2122 & 111.7 & 13.55 \\
\hline SIF REC 3\% & 2129 & 96.7 & 12.65 \\
\hline SIF C 15\% & 2141 & 99.5 & 10.52 \\
\hline
\end{tabular}

It is evident that the addition of fibres leads to the increase in the compressive strength. The same is valid also for the flexural tensile strength with exception of the polypropylene fibres/concrete composite.

Dynamic compression tests were conducted using the striker impact velocity about $25 \mathrm{~m} / \mathrm{s}$. Typical incident stress pulse, $\sigma_{\mathrm{I}}$, is shown in the Fig.3. Time history of the transmitted stress pulse is displayed in the Fig.2a. Specimens of the all tested materials were fractured. The values of the dynamic compressive strength (average from five specimens) are displayed in the Figs. $2 b$.

It is evident that the compressive strength is rate sensitive. Their values obtained at the dynamic loading are higher than those at the quasi-static loading. This increase can be described using of the dynamic increasing factor, DIF $=\sigma_{\mathrm{d}} / \sigma_{\mathrm{c}}$. The values of DIF are presented at the right part of the Fig.2c. The results show that the most sensitive to the increase in the loading rate is concrete without any fibres. The addition of the fibres leads to the decrease in this sensitivity.

In the Fig. 3 typical experimental record of the stress pulses during dynamic tensile splitting test are shown. The details of the fracture of the specimen during the Brazilian test are documented in Fig.4. In the upper raw of this figure, the starting position and the initial crack is shown $(36.5 \mu \mathrm{s}$.). Next frame shows its development in time $43.75 \mu \mathrm{s}$ after the impact and in the last frame the fracture of the specimen after $62.5 \mu$ s after the impact is documented. 


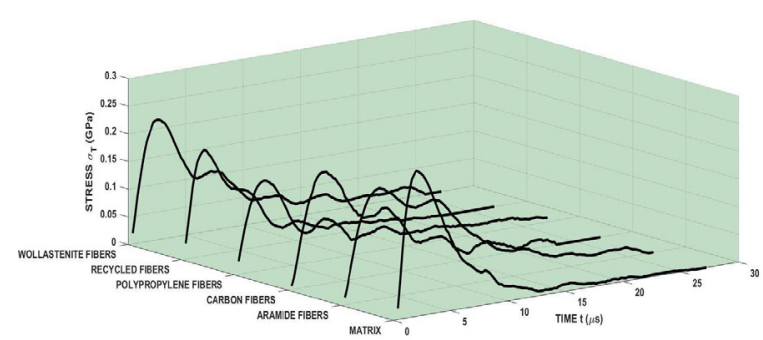

Fig. 2a. Transmitted stress pulses for single materials.

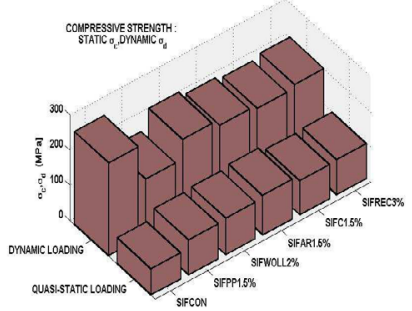

Fig. 2b Dynamic and quasi-static compressive strength.

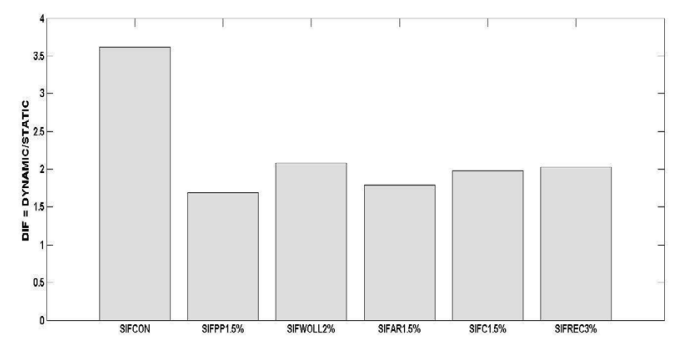

Fig. 2c. Dynamic increase in the compressive strength.
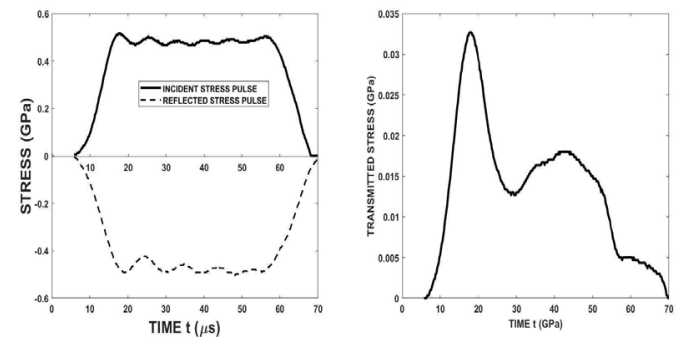

Fig. 3. Experimental record of the stress pulses during the dynamic tensile splitting test.(Aramid fibres/concrete composite).

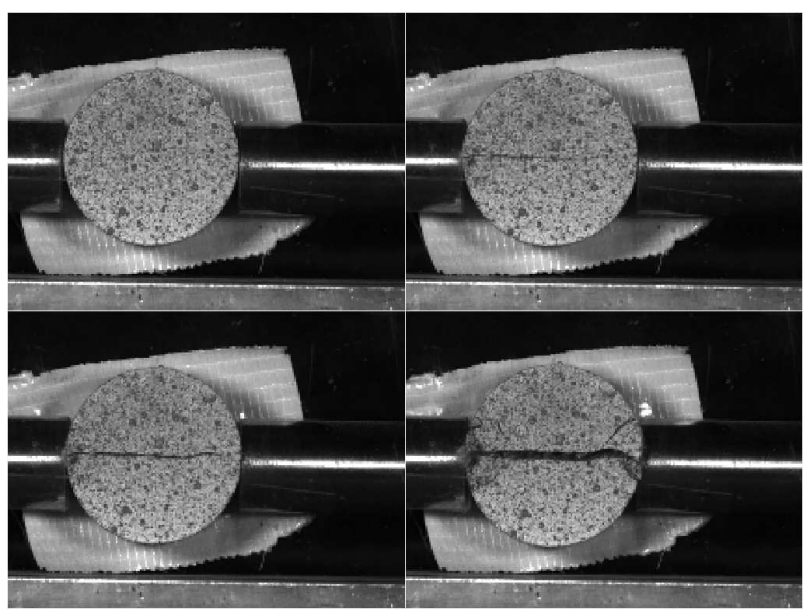

Fig. 4. Development of fracture during the Brazilian test. (aramide fibres reinforced concrete).

Detailed analysis of the results of the high speed camera shows that the beginning of the specimen fracture corresponds to the maximum of the transmitted stress pulse $\sigma_{\mathrm{T}}$. The next fragmentation of the specimen is illustrated in the Fig.5. The fracture pattern is different for different materials - see Fig.6. The frames were captured at the same time. The concrete with polypropylene fibres remains undamaged. The damage was observed after $133 \mu \mathrm{s}$.

The analysis of the experimental data obtained during the Brazilian test revealed that the specimen dimensions had no influence on the values of the tensile strength. Results of the both quasi-static and dynamic tensile strengths are shown in the Fig7a. It is evident that the dynamic loading leads to significant increase in the tensile strength. This increase is described using of DIF $=$ dynamic tensile strength/static tensile strength - see Fig.7b. Similarly as in the case of the compressive strength the effect of the fibre reinforcement is different at quasi - static and dynamic loading.

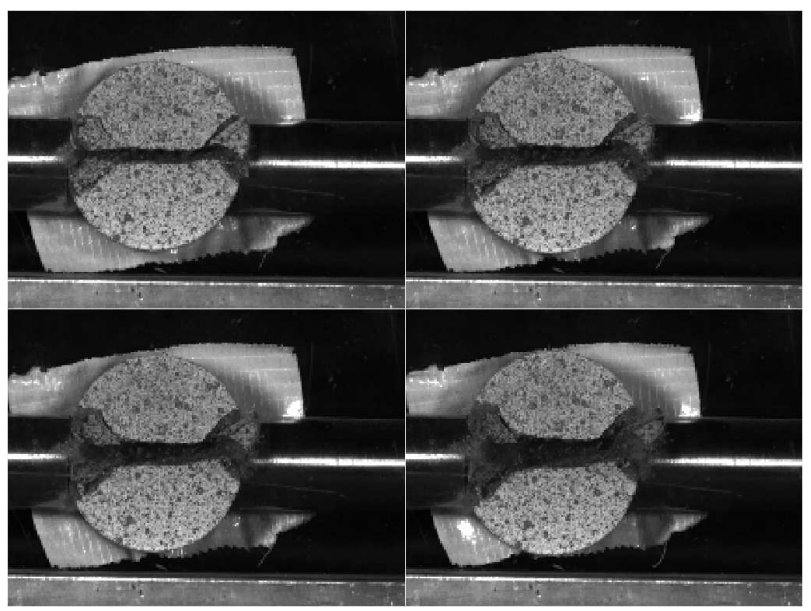

Fig. 5. Fragmentation of the specimen (aramide fibres reinforced concrete). 


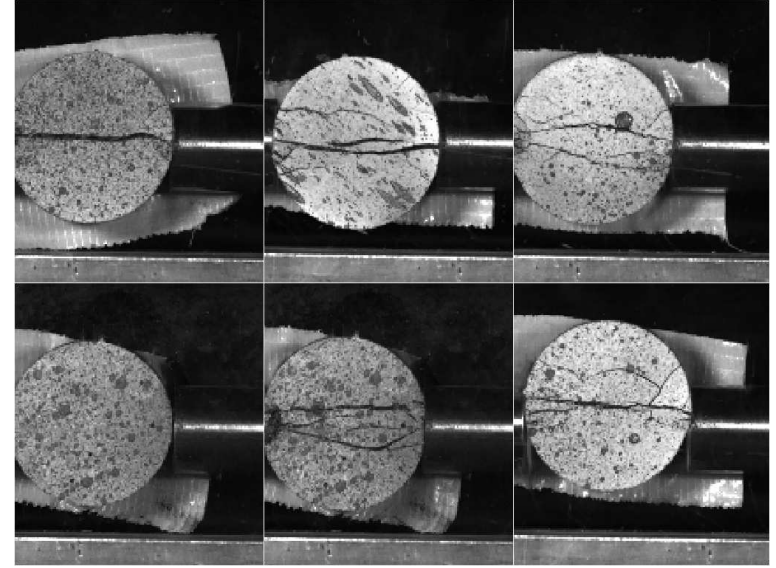

Fig. 6. Fracture of the specimens of different composites after $62.5 \mu \mathrm{s}$. The upper figures from the left to right: Aramide fibres, Carbon fibres, Matrix. The lower figures from the left to right: Polypropylene fibres, Wolastonite fibres, Recycled fibres.

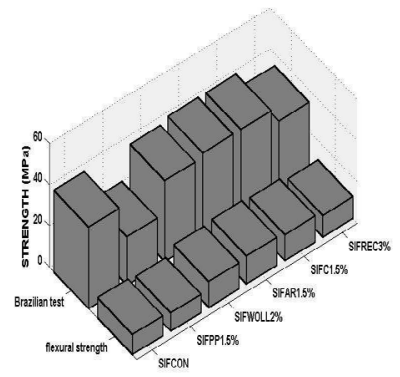

Fig. 7a. Tensile strength of the tested concrete with different fibre reinforcement.

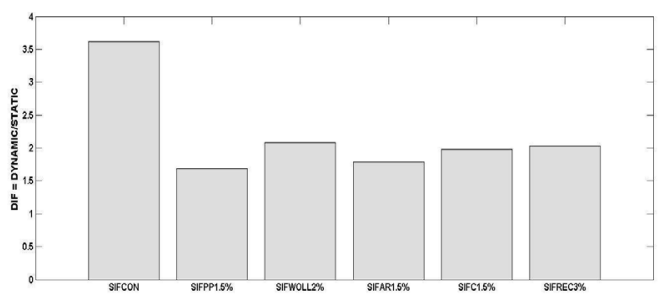

Fig. 7b. Dynamic increase in the tensile strength.

The values of the tensile strength are taken as average values from at least 5 measurements. The values of the tensile strength were achieved at the different time. It means there were different loading rates. The dependence of tensile strength on the loading rate is displayed in the Fig.8.

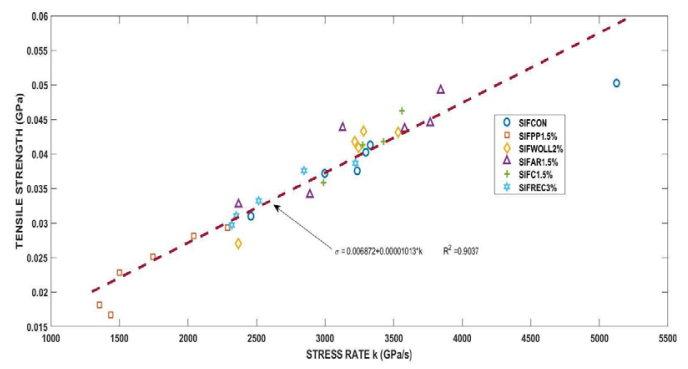

Fig. 8. The effect of the loading rate on the tensile strength at the Brazilian test.
Results show that the loading rates for different materials are different. The average data on the tensile strength and loading rate are presented in the Table 2 .

Table 2. Tensile strength evaluated from the Brazilian test. Data are presented in the form: average value \pm standard deviation.

\begin{tabular}{|c|l|l|}
\hline MATERIAL & Tensile strength & Loading rate \\
\hline SIFCON (Matrix) & $39.58 \pm 6.34$ & $3408.23 \pm 902.67$ \\
\hline SIF PP $1.5 \%$ & $23.38 \pm 5.17$ & $1728.59 \pm 371.25$ \\
\hline SIF woll $\%$ & $39.23 \pm 6.90$ & $3129.43 \pm 443.03$ \\
\hline SIF AR $1.5 \%$ & $41.32 \pm 6.48$ & $3263.04 \pm 574.27$ \\
\hline SIF REC $3 \%$ & $41.31 \pm 4.26$ & $3313.25 \pm 244.89$ \\
\hline SIF C $1.5 \%$ & $34.04 \pm 3.95$ & $2651.68 \pm 381.42$ \\
\hline
\end{tabular}

In order to obtain more detailed information on the fracture process during the Brazilian test, some information on the strain development in the specimen is needed. For this purposes strain gauges were conventionally used to measure the deformation in a Brazilian test. As a contact measurement method, strain gauges have advantages such as high sensitivity and low cost; however, they can only measure one or several points at the same time and cannot conduct a full-field deformation measurement. In addition, for non-uniform strain, the effect of the gauge size is not negligible. As an alternative method, some optical non-contact techniques can provide full-field deformation information. Among the optical techniques, the digital image correlation (DIC) method is one of the most powerful optical full-field measurement methods and has been applied in various fields in the past decades $[8,9]$. By measuring the strain with both the laser gap gauge technique and high-speed DIC method, Chen et al. studied the tensile strength of brittle materials [10]. In the given paper, the dynamic displacement and strain fields were processed using the VIC-2D software.

In Fig. 9, an example of the strain distribution in the specimen made from SIFAR $1.5 \%$ composite is displayed. The normal strains, $\mathrm{E}_{\mathrm{xx}}, \mathrm{E}_{\mathrm{yy}}$ and shear strain $\mathrm{E}_{\mathrm{xy}}$ are shown.

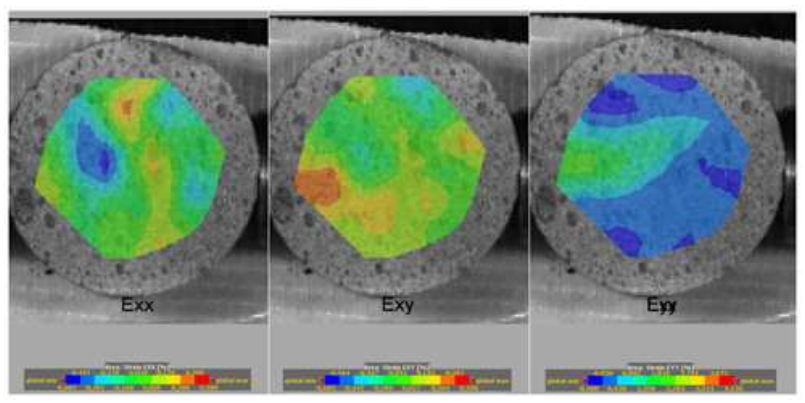

Fig. 9. Example of the strain distribution. $\mathrm{X}$ direction agree with test bar axis, $\mathrm{y}$ direction is normal to this axis.

It is obvious that the distribution of strain is highly non- homogeneous. The fracture development is affected by the stress in the $\mathrm{y}$ direction. The distribution of $\mathrm{E}_{\mathrm{yy}}$ strains in specimens made from different composites is shown in the Fig.10. These frames were taken just before the fracture initiation. It may be seen that the strain distribution exhibits an asymmetry along the y axis. It 
means that there is no equilibrium in the specimen and the validity of the equation ( 3 ) is questionable. It means that the values of the tensile splitting strength may be used for a comparison but it is impossible to use these values as a standard physical quantity. This result suggests that the loading stress pulse of longer time of its duration should be used. Results presented in [4] show that the use the stress pulse duration about $120 \mu$ s leads to the development of the stress equilibrium in the specimen. In this paper the stress pulse duration was about $70 \mu \mathrm{s}$.

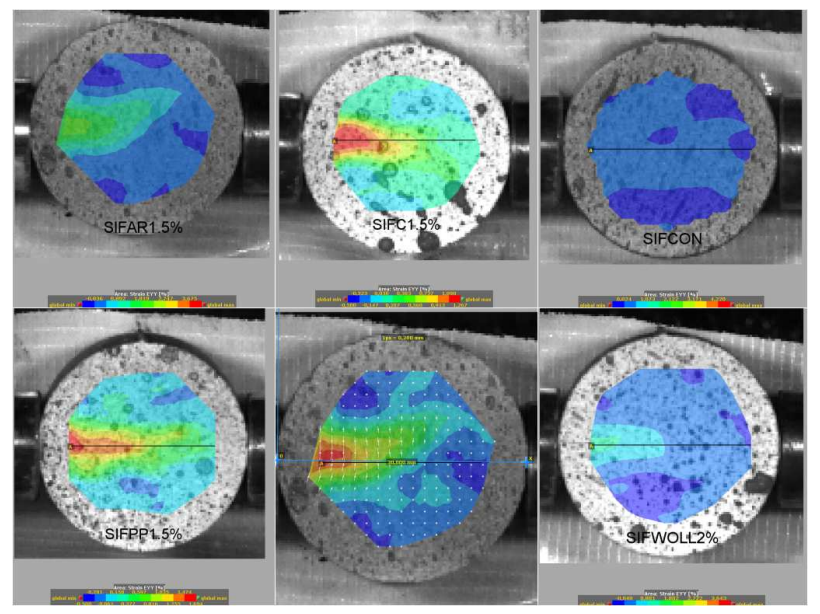

Fig. 10. Distribution of strain in the specimens made from different composites. Figure in the middle of the lower raw shows the coordinate system.

There are remarkable differences in the strain distribution among specimens made from different composites. In order to obtain some more detail information, the dependence of the strain on the distance from the point of specimen loading was evaluated. As a result, the maximum of the strain in the y direction at the moment of fracture initiation was found. The values of this strain, which can be considered as the fracture strain, are plotted in the Fig. 11.

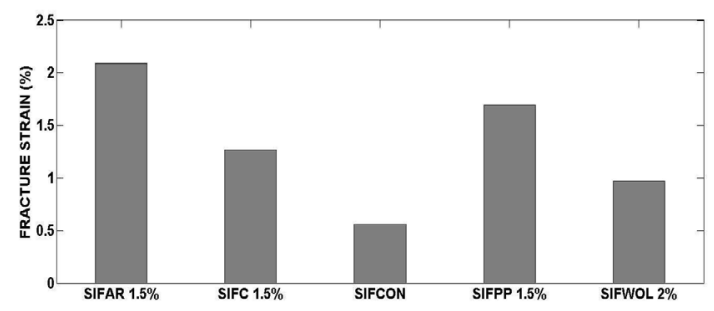

Fig. 11. Strain at the fracture of the specimen.

The maximum of the fracture strain reaches the composite with aramide fibres. The tensile strength is for the most of the tested materials non-increasing function of the fracture strain as it is shown in the Fig.12. The only exception represents the SIFAR $1.5 \%$ composite.

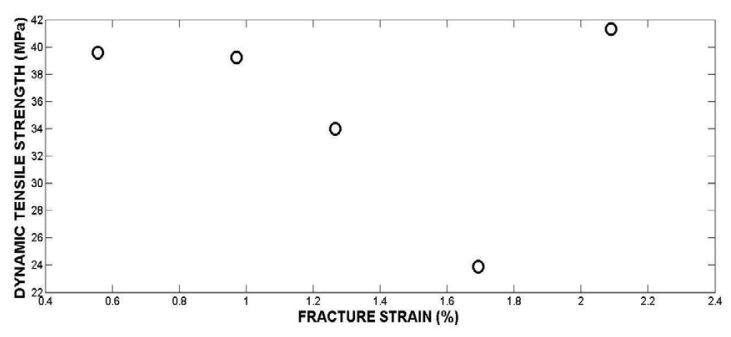

Fig. 12. Tensile strength (average values) vs fracture strain.

The next information on the fracture process can be obtained from the fractographic examination of the fracture surfaces. In the given paper the preliminary results were obtained for the specimens of SIFC1.5\% composite. Fig. 13 shows the fracture surface of these specimens.

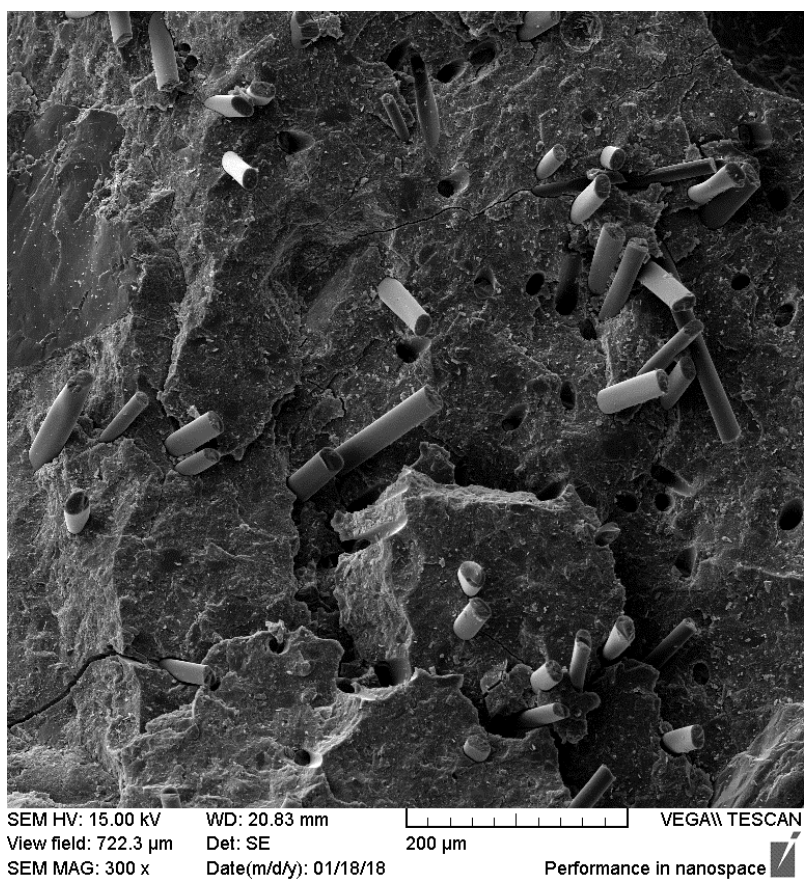

Fig. 13. Example of the fracture surface. See crack in the lower left area.

It may be seen that there are some cracks at the interface between fibres and matrix. Matrix and fibres differs in the acoustic impedances. It means there are conditions for the development of some spall fracture. This phenomenon can be a reason why the fibres lead to the decrease of the strength of the composite in comparison with pure concrete. The verification of this hypothesis needs next detail research which is now in progress.

\section{Concluding remarks}

Static and dynamic properties of concrete reinforced by different fibres were evaluated. The fibre reinforcement of the concrete leads to the improvement of its mechanical properties like the compressive strength and tensile strength. The best efficient seems to be aramide fibres. 
The Hopkinson split pressure bar was used to apply dynamic loading. Results of the compression test show the increase of the dynamic compressive strength in comparison with quasi - static loading. At the same time, the influence of the fibre reinforcement on the compressive strength is quite different from that at the quasi static loading. In the next step, the dynamic Brazilian test was performed. Results of dynamic tensile test show similar features as results of the quasi-static test. Some possible explanation was suggested. Dynamic tensile strengths are rate dependent.

Tensile strength was evaluated using of Eq.(3) which was developed under assumption of the stress equilibrium in the specimen. The DIC was used to conduct a full-field deformation analysis of the disc specimens using images recorded by a high-speed camera. Based on the DIC full-field analysis and stress pulse measurement, stress equilibrium is not satisfied in the dynamic Brazilian test of the tested materials. In order reach a validity of Eq.(3) the loading stress pulse of a sufficient time of duration should be used. Literature data suggest that the duration of the loading pulse should be about $120 \mu$ s.

The authors wish to express their gratitude and sincere appreciation to the authority of The Technology Agency of the Czech Republic, project No. TA02000162 for financial support.

\section{References}

1. A.Prakash,S.M.Srinivasen,A.Rama Mohan Rao. Materials and Design 83,164 (2015)

2. M.Drdlova,M.Frank,J.Buchar,R.Ridky,F.Soukal. .Cellular Polymers 35,143 (2016)

3. Yubin Lu,Shuisheng Yu,Yong Chai.EPJ WEB of Conferences 94,01008 (2015)

4. J.J.Chen,B.Q.Guo,H.B.Lin,P.W.Chen Strain 50,563 (2014)

5. Y.X.Zhou,H.B.Li. Int.J.Rock Mech.Min.Sci 37,861 (2000)

6. S.M.Dong, ,K. Xia, S. Huang, Tubing Yin. Rate dependence of the tensile and flexural strengths of glass-ceramic Macor. J. Mater. Sci. 46, 394 (2011)

7. J.R.Antoun, J. R. ,A.M. Rajendran, Proceedings of the Topical Conference on Shock Compression of Condensed Matter. Elsevier, Amsterdam: 501 (1992)

8. F.Yan, , X.T.Feng, ,R. Chen, R. Rock Mech. Rock. Eng. 45, 341 (2012)

9. W.H. 9.W.H. Peters,,W.F. Ranson,W. F. Opt. Eng. 21, 427(1982)

10. R.Chen, F., Dai, F. Lu, Exp. Mech. 53, 1153-1159 